\title{
Continuous reformulation of binary variables, revisited $^{\star}$
}

\author{
Leo Liberti \\ LIX CNRS , École Polytechnique, Institut Polytechnique de Paris, 91128 Palaiseau, \\ France liberti@lix.polytechnique.fr
}

\begin{abstract}
We discuss a class of tightly feasible MILP for which branchand-bound is ineffective. We consider its hardness, evaluate the probability that randomly generated instances are feasible, and introduce a heuristic solution method based on the old idea of reformulating binary variables to continuous while introducing a linear complementarity constraint. We show the extent of the computational advantage, under a time limit, of our heuristic with respect to a state-of-the-art branch-andbound implementation.
\end{abstract}

Keywords: Reformulation, infeasible, market split, market share, hard instances.

\section{Introduction}

In this paper we consider the following Mixed-Integer Linear Program (MILP):

$$
\left.\begin{array}{rl}
\min \sum_{h \leq p} y_{h} \\
Q y=x \\
A x \leq b \\
x^{L} \leq x \leq x^{U} \\
y \in\{0,1\}^{p}
\end{array}\right\}
$$

where $Q=\left(q_{j h}\right)$ is an $n \times p$ real matrix, $A=\left(a_{i j}\right)$ is an $m \times n$ real matrix, $b \in \mathbb{R}^{m}, x$ is a vector of $n$ continuous decision variables, and $y$ is a vector of $p$ binary decision variables.

Eq. (1) was brought to my attention by a colleague, as an interesting "core" of a much more complicated formulation concerning a Hydro Unit Commitment (HUC) problem arising at Électricité de France (EDF) 6]. Although Eq. (1) is not directly related to HUC, my colleague and her co-workers at EDF have identified Eq. (1) as the source of frequent feasibility issues they experienced while solving their HUC, which they discuss in [21, but along different lines than the present paper.

\footnotetext{
* This work was partially supported by: the PGMO grant "Projet 2016-1751H"; a Siebel Energy Institute Seed Grant; the European Union's Horizon 2020 research and innovation programme under the Marie Sklodowska-Curie grant agreement $n$. 764759 ETN "MINOA".
} 
The binary variables $y$ in Eq. (1) model some on/off controls along a discretized time line. The controls influence (through the equations $Q y=x$ ) some physical quantities $x$ that are constrained to lie in $\left[x^{L}, x^{U}\right]$. The decision maker seeks the smallest number of controls that need to be switched on in order for the physical constraints on $x$ to be feasible. According to [21, however, it is difficult to satisfy the constraints of Eq. (1), at least in the EDF instances. In particular, the authors detail the efforts of solving Eq. (1) with common MILP solution techniques, such as the Branch-and-Bound (BB) solver CPLEX [12, which would normally be considered the best solution method for such problems.

At first sight, the MILP in Eq. (1) may not strike the reader as a particularly "nasty" problem, insofar as structure goes. The infeasibility issues arise because the instances solved at EDF enforce very tight bounds $\left[x^{L}, x^{U}\right]$ on $x$ - sometimes requiring that $x^{L}=x^{U}$ (which occurs in run-of-the-river reservoirs). Note that the constraints $A x \leq b$ in Eq. (1) are supposed to encode "the rest of the problem" (which is quite extensive, and may involve more decision variables than just $x$ ). In a private communication [6, I was told that the infeasibilities were mostly related to the problem in Eq. (2) below, obtained as a relaxation of Eq. (1) by shedding the technical constraints. Accordingly, Eq. (2) will be our problem of interest in the rest of this paper.

$$
\left.\begin{array}{rl}
\min \sum_{h \leq p} y_{h} \\
Q y & =x \\
x^{L} \leq x \leq x^{U} \\
y & \in\{0,1\}^{p} .
\end{array}\right\}
$$

Although in this paper we focus exclusively on feasibility, the objective function is discussed in 23. The choice of limiting our attention to Eq. (2) is also due to confidentiality issues: the EDF instances for the original problem could not be made available to me. I therefore worked with instances generated randomly from Eq. (2).

This paper makes two contributions: a theoretical one about the probability of generating feasible/infeasible random instances of the problem in Eq. (2); and a computational one about a heuristic method for solving it.

From the methodological point of view, we leverage an observation made in 7] about Linear Complementarity Programming (LCP) reformulations of tightly constrained MILPs in binary variables: they often (heuristically) lead to exact, or almost exact, solutions. We discuss the computational and empirical hardness of Eq. (2) and present some solution methodologies. We focus on a specific one based on an LCP reformulation, which we also test computationally.

The rest of this paper is organized as follows. Sect. 2 provides a very short literature review. In Sect. 3 we discuss the computational complexity of our problem. In Sect. 4 we consider the difficulty of the problem in terms of the probability of a random instance being feasible in function of the bounds $\left[x^{L}, x^{U}\right]$. In Sect. 5 we discuss some unusual solution methods that are not based on BB. 


\section{Short literature review}

As mentioned above, the original inspiration for this paper was the HUC problem, although the paper itself is not about HUC (see 23. for more information about HUC). This paper is actually about Eq. (2), specifically in the case where $\left\|x^{L}-x^{U}\right\|_{2}$ is small, or even zero, and the consequent infeasibility issues observed in popular MILP solvers even when instances are feasibile. As already noted, the feasibility issues we study in this paper (quite independently of HUC) have been addressed within the HUC context in [21, Sect. 5], mostly through bound constraint relaxation by means of variables whose sum is minimized.

When $x^{L}=x^{U}$, and without an objective function, Eq. (2) reduces to Eq. (3) (see below), which is a well-known case of difficult Binary Linear Program (BLP), i.e. the so-called market split [5] (a.k.a. "market share"). In the literature, pure feasibility BLPs such as Eq. (3) have been solved by means of a basis reduction algorithm proposed in [1, which also targets its "natural" optimization version minimizing a sum of slack variables added to the equations. Solving Eq. (3) with an arbitrary objective function is outside of the scope of the basis reduction algorithm of 2] (used in [1]), although of course bisection search of the feasibility algorithm of 2 is always available.

We noted that our purpose in this paper is to solve Eq. (2) by means of an LCP reformulation. This is more unusual than the converse (i.e. solving LCPs by means of a MILP reformulations), since state-of-the-art solver technology is better for MILP than for nonconvex Nonlinear Programming (NLP), which includes LCP. A MILP reformulation of the LCP was presented in [19, Thm. 3.1]. This reformulation has been used many times in the literature, and is now part of the Mathematical Programming (MP) "folklore".

Reformulating MILPs to LCPs is more unusual but of course not unheard of: as already stated, we took our methodological inspiration from [7, which reformulates a binary variable vector $y \in\{0,1\}^{p}$ exactly by means of the addition of a nonlinear constraint $\sum_{h \leq p} y_{h}\left(1-y_{h}\right)=0$ to the formulation. This is one of the oldest tricks in MP: to name a few citations, 20[18 add $\sum_{h \leq p} y_{h}\left(1-y_{h}\right)$ as a penalty to the objective, while [17] proposes an alternating heuristic based on $\sum_{h \leq p} y_{h}\left(1-y_{h}\right) \leq \delta$, where $\delta$ is reduced at each iteration.

\section{Hardness}

Just how hard is the problem in Eq. (2)? We consider the following set of linear equations in binary variables $y \in\{0,1\}^{p}$ :

$$
\forall j \leq n \quad \sum_{h \leq p} q_{j h} y_{h}=\bar{x}_{j}
$$

where $\bar{x}=x^{L}=x^{U}$. We assume $Q, \bar{x}$ are rational. We stress that fixing the variables $x$ to a fixed constant $\bar{x}$ appears to be an important practical case [6] in the motivating application. 
First, we consider the case $n=1$, i.e. (3) consists of just one equation. Then Eq. (3) is a rational equivalent of the Subset Sum problem [9, §SP13], with instance given by $(Q, \bar{x})$. The fact that $Q, \bar{x}$ are rational obviously does not change the problem: it suffices to rescale all data by the minimum common multiple of all the denominators. This problem is weakly NP-complete: it can be solved in pseudopolynomial time by a dynamic programming algorithm 9 . The Wikipedia entry for SUBSET SuM also states that the difficulty of solving this problem depends on the number of variables $p$ and the number of bits necessary to encode $Q$ : if either is fixed, the problem becomes tractable. It is well known that Integer Linear Programs (ILP) with a fixed number of variables $p$ can be solved in polynomial time [15].

In this paper, we are interested in the case where $p$ is not fixed, whereas $n$ might be fixed or not. The case with $n$ fixed is relevant for the motivating application, as the operational points $x_{j}$ on which the constraints $x_{j} \in\left[x_{j}^{L}, x_{j}^{U}\right]$ are verified could be given by the (fixed) physical properties of the considered hydro valley. We are not aware of results in ILP complexity with a fixed number of constraints but non-fixed number of variables, however.

If neither $n$ nor $p$ is fixed, we note that there is a natural reduction from SAT to a version of Eq. (3) where $Q$ has entries in $\{-1,0,1\}$, which shows that solving Eq. (3) is strongly NP-complete (by inclusion with respect to $Q$ ). Again by inclusion (with respect to $x^{L}, x^{U}$ ), Eq. (2) is also NP-complete.

Eq. (2) is one of those cases when complexity proofs by inclusion are not quite satisfactory. The empirical hardness of solving Eq. (2) obviously decreases as the bounds $\left[x^{L}, x^{U}\right]$ grow farther apart (if $x^{L}=-\infty$ and $x^{U}=\infty$ any solution of Eq. (2) is trivially feasible). A more convincing complexity proof should take the width parameter $W=\max _{j \leq n}\left(x_{j}^{U}-x_{j}^{L}\right)$ into account, too. In Sect. 4 below we attempt to provide a more appropriate hardness measure in terms of the probability of achieving feasibility in a randomly generated instance.

\section{Likelihood of approximate feasibility}

In this section we consider the probability that uniformly sampled instances of Eq. (3) and Eq. (2) are (almost) feasible.

\subsection{The Irwin-Hall distribution}

Consider Eq. (3). For each $j \leq n$ and $h \leq p$ we assume that $q_{j h}$ is sampled from a random variable $\tilde{Q}_{j h}$ uniformly distributed in $[0,1]$. For a given $y \in\{0,1\}^{p}$, let

$$
\hat{Q}_{j}=\sum_{\substack{h \leq p \\ y_{h}=1}} \tilde{Q}_{j h} .
$$

We intend to derive an expression, which depends on $x^{L}$ and $x^{U}$, of the probability that a uniformly sampled instance of Eq. 22 is feasible. To do that, we first look at the case $\bar{x}=x^{L}=x^{U}$ and tackle instances where the cardinality of 
the support of $y$ is fixed to a given integer $K$, i.e. $\sum_{h \leq p} y_{h}=K$. For all $j \leq n$, the corresponding random variable is

$$
\hat{Q}_{j}^{K}=\sum_{\substack{h \leq p \\ \sum_{h} y_{h}=K}} \tilde{Q}_{j h}
$$

i.e. the sum of $K$ i.i.d. uniform random variables on $[0,1]$.

For any given $j \leq n$, the distribution of $\hat{Q}_{j}^{K}$ is known as the Irwin-Hall distribution [11. Its mean is $K / 2$ and its variance is $K / 12$. It can also be shown that for $K>1$ the probability distribution function (PDF) of $\hat{Q}_{j}^{K}$ attains a strict (local) maximum at the mean $K / 2$. The cumulative distribution function $(\mathrm{CDF})$ is

$$
F_{K}(x)=\frac{1}{K !} \sum_{k=0}^{\lfloor x\rfloor}(-1)^{k}\left(\begin{array}{c}
K \\
k
\end{array}\right)(x-k)^{K} .
$$

By Eq. (4), for any $j \leq n$ the probability of $\hat{Q}_{j}^{K}$ taking values between $x_{j}^{L}$ and $x_{j}^{U}$ is $F_{K}\left(x_{j}^{U}\right)-F_{K}\left(x_{j}^{L}\right)$, a quantity we shall denote by $\gamma_{j}^{K}\left(x^{L}, x^{U}\right)$, or $\gamma_{j}^{K}$ when no ambiguity can ensue.

\subsection{Feasibility for $n=1$}

If we fix $n=1$, understanding the distribution of $\hat{Q}_{1}^{K}$ would allow us to glance at some uniformly generated input data, $(Q, \bar{x})$, and get an idea of the likelihood of a given binary vector $y$ with $K$ nonzeroes yielding the given value $\bar{x}_{1}$.

We first want to make a qualitative statement to the effect that instances where $\left[x_{1}^{L}, x_{1}^{U}\right]$ contains the mean $K / 2$ are likely to be easier than those that do not.

Lemma 4.1. There exists a value $0<r<K$ such that, from $r$ units away from the mean, the tails of the probability density function $(P D F)$ of $\hat{Q}_{1}^{K}$ converge to zero exponentially fast.

Proof. First, we argue about the right tail. Trivially, since $\tilde{Q}_{1 h}$ is uniformly sampled in $[0,1]$ for each $h \leq p$, we have $\mathbb{P}\left(\hat{Q}_{1}^{K} \geq K\right)=0$. We now have to argue the negative exponential convergence in some sub-range of $[K / 2, K]$ including the right end-point $K$. By [14, Thm. 7.5], we have

$$
\mathbb{P}\left(\hat{Q}_{\geq}^{K} \frac{K}{2}+r\right) \leq e^{-\frac{K}{2} g\left(\frac{2 r}{K}\right)}
$$

for any $r>0$, where $g(u)=(1+u) \ln (1+u)-u$ for $u \geq 0$. The exponent in the RHS of Eq. (5) is negative as long as:

$$
\left(1+\frac{2 r}{K}\right) \ln \left(1+\frac{2 r}{K}\right)>\frac{2 r}{K}
$$


Trivially, we have that, for all $v>e, v \ln v>v$. So, if $1+\frac{2 r}{K}>e$, it follows that $(1+2 r / K) \ln (1+2 r / K)>1+2 r / K$, which is obviously strictly greater than $2 r / K$. We therefore need $r>K(e-1) / 2$ for the statement to hold, as claimed.

The argument for the left tail follows by symmetry of the PDF, which can be established by considering the distribution of the sum of $K$ uniform random variables over $[-1,0]$.

By Lemma 4.1 the measure of the PDF of $\hat{Q}_{1}^{K}$ is concentrated around $\frac{K}{2}$. This makes it reasonable to expect that instances will be easier as $\bar{x}$ moves towards $\frac{K}{2}$.

Quantitative statements about the probability of generating feasibile instances can be obtained for given values of $K$ and $x_{1}^{L}, x_{1}^{U}$ by evaluating $\gamma_{1}^{K}\left(x^{L}, x^{U}\right)$.

\subsection{Feasibility in the general case}

We now move back to the general case with $n>1$ and bounds $x^{L}, x^{U} \in \mathbb{R}^{n}$ on $x$ as in Eq. (2). Recall that for all $j \leq n$ we defined

$$
\mathbb{P}\left(\exists y \in\{0,1\}^{p} \hat{Q}_{j}^{K} \in\left[x_{j}^{L}, x_{j}^{U}\right] \mid \mathbf{1} y=K\right)=\gamma_{j}^{K} .
$$

Proposition 4.2. The probability that a uniformly sampled instance of Eq. (2) is feasible is:

$$
\mathbb{P}\left(\exists y \in\{0,1\}^{p} \text { s.t. } \tilde{Q} y \in\left[x^{L}, x^{U}\right]\right) \leq \frac{1}{2^{p}} \sum_{K \leq p}\left(\begin{array}{c}
p \\
K
\end{array}\right)\left(1-\prod_{j \leq n}\left(1-\gamma_{j}^{K}\right)\right) .
$$

Proof. The probability that there exists $y$ with support cardinality $K$ that satisfies all of the constraints is

$$
\begin{aligned}
& \mathbb{P}\left(\exists y \in\{0,1\}^{p} \hat{Q}^{K} \in\left[x^{L}, x^{U}\right] \mid \mathbf{1} y=K\right)= \\
= & 1-\mathbb{P}\left(\forall y \in\{0,1\}^{p} \hat{Q}^{K} \notin\left[x^{L}, x^{U}\right] \mid \mathbf{1} y=K\right)= \\
= & 1-\mathbb{P}\left(\forall y \in\{0,1\}^{p} \exists j \leq n \hat{Q}_{j}^{K} \notin\left[x_{j}^{L}, x_{j}^{U}\right] \mid \mathbf{1} y=K\right) \leq \\
\leq & 1-\mathbb{P}\left(\exists j \leq n \forall y \in\{0,1\}^{p} \hat{Q}_{j}^{K} \notin\left[x_{j}^{L}, x_{j}^{U}\right] \mid \mathbf{1} y=K\right) \leq \\
\leq & 1-\mathbb{P}\left(\forall j \leq n \forall y \in\{0,1\}^{p} \hat{Q}_{j}^{K} \notin\left[x_{j}^{L}, x_{j}^{U}\right] \mid \mathbf{1} y=K\right)= \\
= & 1-\mathbb{P}\left(\bigwedge_{j \leq n} \forall y \in\{0,1\}^{p} \hat{Q}_{j}^{K} \notin\left[x_{j}^{L}, x_{j}^{U}\right] \mid \mathbf{1} y=K\right)= \\
= & 1-\prod_{j \leq n} \mathbb{P}\left(\forall y \in\{0,1\}^{p} \hat{Q}_{j}^{K} \notin\left[x_{j}^{L}, x_{j}^{U}\right] \mid \mathbf{1} y=K\right)= \\
= & 1-\prod_{j \leq n}\left(1-\mathbb{P}\left(\neg \forall y \in\{0,1\}^{p} \hat{Q}_{j}^{K} \notin\left[x_{j}^{L}, x_{j}^{U}\right] \mid \mathbf{1} y=K\right)\right)= \\
= & 1-\prod_{j \leq n}\left(1-\mathbb{P}\left(\exists y \in\{0,1\}^{p} \hat{Q}_{j}^{K} \in\left[x_{j}^{L}, x_{j}^{U}\right] \mid \mathbf{1} y=K\right)\right)= \\
= & 1-\prod_{j \leq n}\left(1-\gamma_{j}^{K}\right),
\end{aligned}
$$


where the last equality follows by Eq. (6). We can take the union over all possible values of $K \in\{0, \ldots p\}$ by weighing each probability by the probability that a uniformly sampled binary vector $y$ should have support cardinality $K$, i.e. $\left(\begin{array}{l}p \\ K\end{array}\right) / 2^{p}$, which yields Eq. (7), as claimed.

For example, if $x^{L}=0$ and $x^{U}=p$, then obviously $\gamma_{K}(j)=1$ for each $K \leq p$ and $j \leq n$, which yields $\frac{1}{2^{p}} \sum_{K \leq p}\left(\begin{array}{l}p \\ k\end{array}\right)=1$, as expected. Setting different values of $x^{L}, x^{U}$, Prop. 4.2 allows the computation of the probability of feasibility of the corresponding instance.

\section{Solution methods}

Since Eq. (2) is a MILP, the solution method of choice is the Branch-and-Bound, implemented for example using a state-of-the-art solver such as CPLEX [12. As mentioned in Sect. 1. however, given the difficulty in finding feasible solutions, CPLEX can rarely prune by bound, which means that it shows the brunt of its exponential behaviour. In this section we discuss three heuristic methods, and argue that the method in Sect. 5.1 is the most promising.

\subsection{Relaxation of integrality constraints}

Every BLP

$$
\min \left\{c^{\top} y \mid B y \leq \beta \wedge y \in\{0,1\}^{p}\right\}
$$

can be exactly reformulated to an LCP by replacing the integrality constraints $y \in\{0,1\}^{p}$ by the following:

$$
\begin{array}{r}
0 \leq y \leq 1 \\
z=1-y \\
\sum_{h \leq p} y_{h} z_{h}=0 .
\end{array}
$$

By Eq. (9)-(10) we have $0 \leq z \leq 1$, which implies that every product in Eq. (11) is non-negative, which in turn means that the sum is non-negative. Thus, the sum is zero if and only if every term is zero, so either $y_{h}=0$ or $z_{h}=1-y_{h}=0$, i.e. $y_{h} \in\{0,1\}$ for each $h \leq p$. An equivalent NLP removes the $z$ variables altogether:

$$
\min \left\{c^{\top} y \mid B y \leq \beta \wedge \sum_{h \leq p} y_{h}\left(1-y_{h}\right)=0\right\} .
$$

This provides the following empirically efficient heuristic algorithm for our problem $P$ in Eq. (2):

1. consider the continuous relaxation $\bar{P}$ of $P$;

2. solve $\bar{P}$ in polynomial time, e.g. by the interior point method; 
3. use the solution $x^{\prime}, y^{\prime}$ as a starting point for a local NLP solver on the problem

$$
\min \left\{\sum_{h \leq p} y_{h} \mid Q y=x \wedge x \in\left[x^{L}, x^{U}\right] \wedge \sum_{h \leq p} y_{h}\left(1-y_{h}\right)=0\right\} .
$$

We remark that only Step 3 is crucial: randomly sampling the starting point is also a valid choice, as evidenced by the reasonable success of Multi-Start (MS) methods for global optimization [16].

In practice, we employ a slack variable $s \geq 0$ on the linear complementarity constraint Eq. 11] in Eq. (13):

$$
\left.\forall j \leq n \quad \begin{array}{rl}
\sum_{h \leq p} y_{h}+\eta s & \\
\sum_{h \leq p} q_{j h} y_{h} & =x \\
\sum_{h \leq p} y_{h}\left(1-y_{h}\right) & =s \\
x & \in\left[x^{L}, x^{U}\right] \\
s & \geq 0,
\end{array}\right\}
$$

where $\eta>0$ is a scaling coefficient chosen empirically. The interest of Eq. (14) is that it always provides a solution: even when $s>0$ (and hence Eq. (11) is not satisfied), we can always hope that the (fractional) $y$ variables will be close enough to integrality that a rounding will yield a feasible solution. As shown in Sect. 6, we consider the solution $\left(x^{*}, y^{*}\right)$ where $y^{*}$ is obtained by rounding the $y$ variables, and $x^{*}$ are re-computed as $Q y^{*}$ according to Eq. (3).

\subsection{The case of fixed $n$}

When $n$ is fixed, we can exploit Barvinok's polynomial-time algorithm for systems with a fixed number of homogeneous quadratic equations [3]. We consider the case where $\bar{x}=x^{L}=x^{U}$ (Eq. (3)), together with the constraint $\sum_{h} y_{h}\left(y_{h}-1\right)=0$, which we homogenize by adding a new variable $z$ and noting that $y_{h}^{2}=y_{h}$ for each $h \leq p\left(\right.$ since $\left.y \in\{0,1\}^{p}\right)$ :

$$
\begin{aligned}
\forall j \leq n \quad \sum_{h \leq p} q_{j h} y_{h}^{2} & =\bar{x}_{j} z^{2} \\
\sum_{h \leq p} y_{h}^{2} & =\sum_{h \leq p} y_{h} z .
\end{aligned}
$$

We remark that all variables $y, z$ are now continuous and unconstrained, but if we achieve a feasible solution with $y \geq 0$ then Eq. (16) will necessarily imply $y \in\{0,1\}$ and $z=1$. We deal with the objective function $\min \sum_{h} y_{h}$ by replacing each $y_{h}$ with $y_{h}^{2}$, to obtain

$$
\left.\left.\min \left\{\sum_{h \leq p} y_{h}^{2} \mid \text { Eq. } 15\right)-16\right)\right\}
$$


which we can solve by a bisection method on the objective function value using Barvinok's algorithm as a feasibility oracle. This requires the homogenization of the equation $\sum_{h} y_{h}^{2}=c$, where $c$ is a constant varied by the bisection method. The system passed to Barvinok's algorithm is therefore

$$
\sum_{h \leq p} y_{h}^{2}=c z^{2} \wedge \text { Eq. 15. } 16 \text {. }
$$

After each call to Barvinok's algorithm, the condition $y \geq 0$ must be verified. If it does not hold, then the heuristic stops inconclusively. Otherwise it identifies the optimum of Eq. (2) up to a given $\epsilon>0$ in time $O\left(\log (p / \epsilon) p^{n}\right)$, polynomial when $n$ is fixed.

We chose not to implement this heuristic, for three reasons: (i) as given in [3], Barvinok's algorithm determines whether system of homogeneous quadratic equations have a common root or not, but will not find the root; (ii) it might not be very efficient in case $n$ is fixed but not extremely small; (iii) the derived heuristic does not appear to provide any useful information in case of failure.

\subsection{Relaxing the $[0,1]$ bounds}

We again assume $\bar{x}=x^{L}=x^{U}$ as in Eq. (3). We also assume that $Q$ and $\bar{x}$ have integer components, and relax the $y$ variables to take any integer value rather than just binary.

This setting opens up algorithmic opportunities from the field of linear Diophantine equations. There exist appropriately sized unimodular matrices $L, R$ such that $L Q R$ is a diagonal matrix $D$ where each nonzero diagonal entry divides the next nonzero diagonal entry [13, Thm. 1]. The system $Q y=\bar{x}$ (with $y \in \mathbb{Z}^{p}$ ) can therefore be decomposed into $D z=L \bar{x}$ and $y=R z$. Now, assuming one can find $L, R$, solving $D z=L \bar{x}$ is easy since $D$ is diagonal, and then $y$ can be computed from $R z$. There are algorithms for computing $L, R$ that work in a cubic number of steps in function of $n, p$ [4].

If we step back to the specificities of the real application, $Q$ consists of floating point numbers given to a precision of at least $10^{-6}$ : rescaling would likely yield large integer coefficients, which would probably make the application of solution algorithms for linear Diophantine equations unwieldy. On the other hand, a systematic treatment of classical results about linear Diophantine equations with rational coefficients and unbounded variables is given in [22, Ch. 4]. Imposing bounds on the variables, however, makes the problem hard again [2]. We therefore decided not to implement this method.

\section{Computational results}

We implemented and tested a MS heuristic defined as follows:

1. sample a starting point $x^{\prime} \in \mathbb{R}^{n}, y^{\prime} \in\{0,1\}^{p}$;

2. deploy a local NLP solver from the initial point $\left(x^{\prime}, y^{\prime}\right)$ on Eq. 14, get $y^{*}$; 
3. round the solution $y^{*}$ and compute $x^{*}=Q y^{*}$;

4. if the result $\left(x^{*}, y^{*}\right)$ has a better objective function value than the incumbent (i.e. the best result found so far), update it;

5. repeat until a termination condition holds.

Our tests aim at verifying whether the above algorithm is competitive compared with a BB-based solver. To this goal, we randomly generated two sets of instances $I_{1}, I_{2}$ of Eq. 22), depending on a vector $\bar{x} \in \mathbb{R}^{n}$, over the following parameters:

$-n \in\{1,2,5,10,50,99\}$

$-p \in\{10,50,100,500,999\}$;

- all components of $Q$ uniformly sampled from either $[0,1]$ (if distr $=0$ ) or $[-1,1]$ (if distr $=1$ );

$-x^{L}=\bar{x}-\theta$ and $x^{U}=\bar{x}+\theta$ for $\theta \in\{0,0.05,0.1\}$, and $\bar{x}$ chosen as detailed below depending on $I_{1}$ or $I_{2}$.

The first set $I_{1}$ contains instances that are feasible by construction. This is achieved by defining $\bar{x}$ as follows:

1. sample $y \in\{0,1\}^{p}$ from $p$ Bernoulli distributions with probability $\frac{1}{2}$ (which implies that the average value of $K$ is $\frac{p}{2}$ );

2. for all $j \leq n$ compute $\bar{x}_{j}=\sum_{h \leq p} Q_{j h} y_{h}$.

The second set $I_{2}$ contains instances that are infeasible with some probability: this is achieved by sampling each component of $\bar{x}$ from a uniform distribution on $[0, \mathcal{Q}]$ (if $\operatorname{distr}=0$ ) or $[-\mathcal{Q}, \mathcal{Q}]$ (if distr $=1$ ), where $\mathcal{Q}=\sum_{h \leq p}\left|Q_{j h}\right|$.

We attempted to compute the probability of infeasibility of the instances in $I_{2}$ by means of Prop. 4.2 , but our naive implementation (based on the AMPL modelling language [8]) was only able to compute the probability of the smallest instances in the set, i.e. those with $p=10$ (for any $n$, distr, $\theta$ ). Those instances with $\theta=0$ obviously yield zero probability by definition of $\gamma_{j}^{K}$. The rest are reported in Table 1 .

We then solved the instances in $I_{1}, I_{2}$ by running the BB solver CPLEX 12.6.3 12 and the MS heuristic above on a 4-CPU Intel Xeon X3220 at $2.4 \mathrm{GHz}$ with 8GB RAM running Linux. We chose SNOPT 7.2 as local NLP solver 10 in the MS heuristic. We gave CPLEX the default parameters but set the time limit to 180s of "wall-clock" time (we recall that CPLEX is a parallel solver by default, so the CPU time measured in the experiments is not limited by 180 s but by the actual user CPU time spent, as reported by the operating system).

The performance of $\mathrm{BB}$ solvers on possibly infeasible instances is severely impaired by a lack of feasible solution because no node is ever pruned by bound; the overall effect is more similar to a complete enumeration than to the typically "smart" enumeration carried out by such solvers. To avoid penalizing CPLEX for this reason, we employed a reformulation of Eq. (2) which shifts all the 


\begin{tabular}{|rrr|r|}
\hline \multicolumn{2}{|r}{$n$ distr } & $\theta$ & probability \\
\hline 1 & 0 & 0.05 & 0.0000000 \\
1 & 0 & 0.10 & 0.0000000 \\
1 & 1 & 0.05 & 0.0000000 \\
1 & 1 & 0.10 & 0.0002403 \\
2 & 0 & 0.05 & 0.0002937 \\
2 & 0 & 0.10 & 0.0033951 \\
2 & 1 & 0.05 & 0.0001573 \\
2 & 1 & 0.10 & 0.0001573 \\
5 & 0 & 0.05 & 0.0024276 \\
5 & 0 & 0.10 & 0.0044601 \\
5 & 1 & 0.05 & 0.0020171 \\
5 & 1 & 0.10 & 0.0007822 \\
10 & 0 & 0.05 & 0.0030972 \\
10 & 0 & 0.10 & 0.0060456 \\
10 & 1 & 0.05 & 0.0015705 \\
10 & 1 & 0.10 & 0.0038300 \\
50 & 0 & 0.05 & 0.0034620 \\
50 & 0 & 0.10 & 0.0070301 \\
50 & 1 & 0.05 & 0.0023307 \\
50 & 1 & 0.10 & 0.0034504 \\
99 & 0 & 0.05 & 0.0038649 \\
99 & 0 & 0.10 & 0.0069426 \\
99 & 1 & 0.05 & 0.0014419 \\
99 & 1 & 0.10 & 0.0029586 \\
\hline \multicolumn{2}{|c|}{$a v e r a g e$} & 0.0024981 \\
\hline \multicolumn{4}{|r|}{}
\end{tabular}

Table 1. The smallest (probably) "infeasible instances" of set $I_{2}$ have low probability of being feasible (computed using Prop. 4.2).

infeasibility into slack variables:

$$
\begin{aligned}
& \min _{x, y, s} \sum_{h \leq p} y_{h}+\sum_{j \leq n}\left(s_{j}^{+}+s_{j}^{-}\right) \\
& \forall j \leq n \quad \sum_{h \leq p} q_{j h} y_{h}=x_{j}+s_{j}^{+}-s_{j}^{-} \\
& x^{L} \leq x \leq x^{U} \\
& s^{+}, s^{-} \geq 0 \\
& y \in\{0,1\}^{p},
\end{aligned}
$$

and carried out the same modification on Eq. (14).

The only possible configuration for the MS heuristic is the maximum allowed number $T$ of iterations, which we set to $p$ (the same as the number of binary variables) in order to have the effort depend on the size of the instance.

The results for the feasible instance set $I_{1}$ are presented in Table 2 We report the instance details $\left(n, p\right.$, the distribution type distr, the $\left[x^{L}, x^{U}\right]$ range half-width $\theta$ ), whether each method found a feasible solution (feas $\in\{0,1\}$ ), the sum of the infeasibilities w.r.t. Eq. (3) conerr, computed as

$$
\text { conerr }=\sum_{j \leq n}\left(\max \left(0, x_{j}^{*}-x_{j}^{U}\right)+\max \left(0, x_{j}^{L}-x_{j}^{*}\right)\right),
$$

and the CPU times (CPU). An instance is classifed as feasible (feas = 1) if conerr $<10^{-8}$ (with also, obviously, $y^{*} \in\{0,1\}^{p}$ ). 

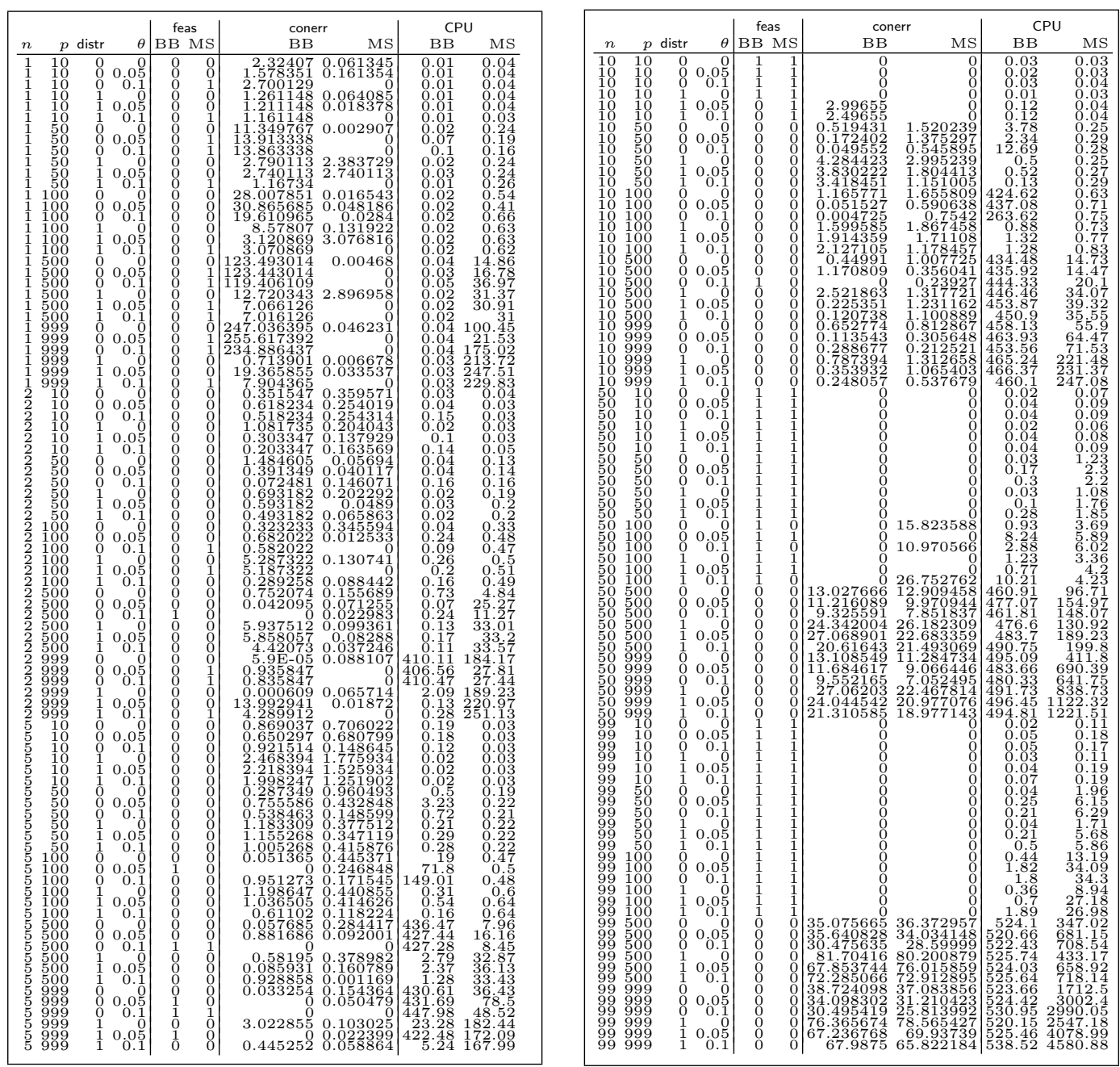

Table 2. Comparative detailed result on the feasible instance set $I_{1}$ with $T=p$ MS iterations.

We remark that we report 6 decimal digits in Table 2 but the computations have been carried out at the machine floating point precision. We report sum, averages and standard deviations for feas, conerr, CPU in Table 3. For lack of space we only report sum, average and standard deviations (rather than complete results) for the results on the possibly infeasible instance set $I_{2}$. It is clear from Tables 3 that continuous optimization techniques are extremely beneficial in finding feasible solutions to tightly constrained MILPs.

One might question whether the superior performance of the MS heuristic is due to the higher CPU time effort of MS w.r.t. CPLEX. To ascertain this, 


\begin{tabular}{l|rr|rr|rr} 
& \multicolumn{2}{|c|}{ feas } & \multicolumn{2}{|c|}{ conerr } & \multicolumn{2}{c}{ CPU } \\
& $\mathrm{BB}$ & $\mathrm{MS}$ & $\mathrm{BB}$ & $\mathrm{MS}$ & $\mathrm{BB}$ & $\mathrm{MS}$ \\
\hline sum & 47 & $\mathbf{5 9}$ & 2270.01 & $\mathbf{9 3 4 . 4 7}$ & $\mathbf{2 3 2 5 4}$ & 32600 \\
avg & 0.261 & $\mathbf{0 . 3 2 8}$ & 12.61 & $\mathbf{5 . 1 9}$ & $\mathbf{1 2 9 . 2}$ & 181.1 \\
stdev & 0.44 & 0.47 & 36.95 & 14.78 & 209.4 & 610.3
\end{tabular}

Table 3. Sums, averages and standard deviations for the instance set $I_{1}$ (Table 2).

\begin{tabular}{l|rr|rr|rr} 
& \multicolumn{2}{|c|}{ feas } & \multicolumn{2}{|c|}{ conerr } & \multicolumn{2}{|c}{ CPU } \\
& $\mathrm{BB}$ & $\mathrm{MS}$ & $\mathrm{BB}$ & $\mathrm{MS}$ & $\mathrm{BB}$ & $\mathrm{MS}$ \\
\hline sum & 0 & $\mathbf{4}$ & 275542.20 & $\mathbf{2 7 4 8 5 8 . 0 3}$ & $\mathbf{2 1 1 . 4 4}$ & 5985.9 \\
avg & 0 & $\mathbf{0 . 0 2 2}$ & 1530.79 & $\mathbf{1 5 2 6 . 9 9}$ & $\mathbf{1 . 1 7}$ & 33.26 \\
stdev & 0 & 0.148 & 3827.90 & 3831.38 & 8.93 & 73.71
\end{tabular}

Table 4. Sums, averages and standard deviations for the instance set $I_{2}$.

we re-ran the experiments with the CPU time of the MS heuristic capped at 3 minutes. Table 5 reports on the sums, averages and variances of results obtained on both $I_{1}$ and $I_{2}$ this way. We remark that the results on $I_{1}$ in Table 5 are

\begin{tabular}{l|rrr|rrr} 
& \multicolumn{3}{|c|}{ Feasible (set $\left.I_{1}\right)$} & \multicolumn{3}{|c}{ Possibly infeas. (set $\left.I_{2}\right)$} \\
& feas & conerr & CPU & feas & conerr & CPU \\
\hline sum & 81 & 916.08 & 8395.9 & 6 & 274834.36 & 3916.4 \\
avg & 0.45 & 5.09 & 46.64 & 0.03 & 1526.86 & 21.758 \\
stdev & 0.50 & 15.33 & 93.22 & 0.18 & 3831.52 & 37.88
\end{tabular}

Table 5. Sums, averages and std. dev. for MS on $I_{1}, I_{2}$ capped at $180 \mathrm{~s}$ CPU time.

actually better than those in Table 3. This is just due to the stochastic nature of the MS heuristic, and to the fact that good solutions are identified early on in the search. Overall, we conclude that the CPU time taken by MS is not the reason for its advantage w.r.t. BB.

\section{Conclusion}

We explored the old idea of replacing binary variables by continuous ones bounded by $[0,1]$ and a linear complementarity constraint. For tightly constrained MILPs similar to market share instances, we show that a simple multi-start approach is superior (under a time limit) to a state-of-the-art branch-and-bound solver. 


\section{References}

1. K. Aardal, R. Bixby, C. Hurkens, A. Lenstra, and J. Smeltink. Market split and basis reduction: Towards a solution of the Cornuéjols-Dawande instances. In G. Cornuéjols, R. Burkard, and G. Woeginger, editors, Integer Programming and Combinatorial Optimization, volume 1610 of LNCS, pages 1-16, Berlin, 1999. Springer.

2. K. Aardal, C. Hurkens, and A. Lenstra. Solving a system of linear Diophantine equations with lower and upper bounds on the variables. Math. Oper. Res., 25(3):427-442, 2000.

3. A. Barvinok. Feasibility testing for systems of real quadratic equations. Discret. Comput. Geom., 10:1-13, 1993.

4. G. Bradley. Algorithms for Hermite and Smith normal matrices and linear Diophantine equations. Math. Comput., 25(116):897-907, 1971.

5. G. Cornuéjols and M. Dawande. A class of hard small 0-1 programs. In R. Bixby, E. Boyd, and R. Ríos-Mercado, editors, Integer Programming and Combinatorial Optimization, volume 1412 of LNCS, pages 284-293, Berlin, 1998. Springer.

6. C. D'Ambrosio. Personal communication, 2017.

7. L. Di Giacomo, G. Patrizi, and E. Argento. Linear complementarity as a general solution method to combinatorial problems. INFORMS J. on Comput., 19(1):7379, 2007.

8. R. Fourer and D. Gay. The AMPL Book. Duxbury Press, Pacific Grove, 2002.

9. M. Garey and D. Johnson. Computers and Intractability: a Guide to the Theory of NP-Completeness. Freeman and Company, New York, 1979.

10. P. Gill. User's guide for SNOPT version 7.2. Systems Optimization Laboratory, Stanford University, California, 2006.

11. P. Hall. The distribution of means for samples of size $n$ drawn from a population in which the variate takes values between 0 and 1 , all such values being equally probable. Biometrika, 19(3/4):240-245, 1927.

12. IBM. ILOG CPLEX 12.10 User's Manual. IBM, 2020.

13. F. Lazebnik. On systems of linear Diophantine equations. Math. Mag., 69(4):261$266,1996$.

14. M. Ledoux. The concentration of measure phenomenon. Number 89 in Mathematical Surveys and Monographs. AMS, Providence, RI, 2005.

15. H. Lenstra. Integer programming with a fixed number of variables. Math. Oper. Res., 8(4):538-548, 1983.

16. M. Locatelli and F. Schoen. Random linkage: a family of acceptance/rejection algorithms for global optimization. Math. Program., 85(2):379-396, 1999.

17. C. Lopez and J. Beasley. A note on solving MINLP's using formulation space search. Optim. Lett., 8:1167-1182, 2014.

18. W. Murray and K.-M. Ng. An algorithm for nonlinear optimization problems with binary variables. Comput. Optim. Appl., 47:257-288, 2010.

19. P. Pardalos and J. Rosen. Global optimization approach to the linear complementarity problem. SIAM J. Sci. Stat. Comput., 9(2):341-353, 1988.

20. M. Raghavachari. On connections between zero-one integer programming and concave programming under linear constraints. Oper. Res., 17(4):680-684, 1969.

21. Y. Sahraoui, P. Bendotti, and C. D'Ambrosio. Real-world hydro-power unitcommitment: dealing with numerical errors and feasibility issues. Energy, 184:91104, 2019.

22. A. Schrijver. Theory of Linear and Integer Programming. Wiley, Chichester, 1986. 
23. R. Taktak and C. D'Ambrosio. An overview on mathematical programming approaches for the deterministic unit commitment problem in hydro valleys. Energy Syst., 8(1):57-79, 2017. 